Article

\title{
On Maximal Homogeneous 3-Geometries- A Polyhedron Algorithm for Space Tilings
}

\author{
István Prok \\ Department of Geometry, Institute of Mathematics, Budapest University of Technology and Economics; \\ P.O. Box 91, H-1521 Budapest, Hungary; prok@math.bme.hu; Tel.: +36-1-463-2644
}

Received: 22 December 2017; Accepted: 27 February 2018; Published: 6 March 2018

\begin{abstract}
In this paper we introduce a polyhedron algorithm that has been developed for finding space groups. In order to demonstrate the problem and the main steps of the algorithm, we consider some regular plane tilings in our examples, and then we generalize the method for 3-dimensional spaces. Applying the algorithm and its computer implementation we investigate periodic, face-to-face, regular polyhedron tilings in 3-spaces of constant curvature and of the other homogeneous 3-geometries, too. We summarize and visualize the most important results, emphasizing the fixedpoint-free space groups which determine 3-dimensional manifolds.
\end{abstract}

Keywords: discrete transformation groups; polyhedron algorithms; manifolds

\section{Introduction}

In some 2-dimensional examples we introduce the basic problem, examining how a regular polygon can be a fundamental domain of a plane crystallographic group. We also develop a combinatorial algorithm for the problem, and we extend the investigation onto the spherical and hyperbolic plane too. The results, among them some new ones, are listed in Table 1 (see in Section 3). Then we generalise the combinatorial algorithm for 3-dimensional problems. Working on the incidence structure of regular polyhedra this algorithm can find the discrete transformation groups of spaces of constant curvature which act simply transitively on regular polyhedron tilings. The fixed-point-free transformation groups lead to 3-dimensional manifolds. The old and new result are summarised in Table 2 (see in Section 5).

As the titles show, this paper is in a close connection with another paper [1] of this volume. For example in [1] on the basis of projective interpretation of the eight Thurston geometries we introduce also an infinite series of hyperbolic manifolds derived not from a regular polyhedron.

\subsection{Introductory Examples}

Let us consider a number of marked tiles, as so-called asymmetric units, containing the same figure (or its mirror image). For example, we draw a hand (right hand or left hand) taking a pen into the square-form tiles. Then we tile the plane with them periodically creating a so called fundamental tiling. This means that an arbitrary tile is a fundamental domain of the symmetry group of the tiling. In other words, the images of the chosen tile under the transformations of the symmetry group fill the plane without gaps and overlaps, creating the tiling. Our aim is typically to find all the essentially different fundamental tilings with our marked tiles. The symmetry group of such a tiling acts simply transitively on the tiles (namely, there is exactly one transformation mapping a tile onto another one) forming a subgroup of the complete symmetry group of the (non-marked) square tiling.

In this way the symmetry group of the first tiling in Figure 1a is the plane crystallographic group p1 (No. 1) generated by two independent translations. Similarly, the symmetry group of the second tiling in Figure $1 \mathrm{~b}$ is $\mathbf{p} 2$ (No. 2). Here a vertical translation and two half-turns (around the 
midpoints of the vertical edges of a tile) generate the group. (Of course, half-turns around two vertices and horizontal translation also occurs as the composition of two half-turns is a translation.)

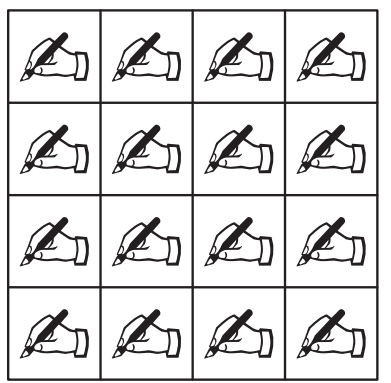

(a)

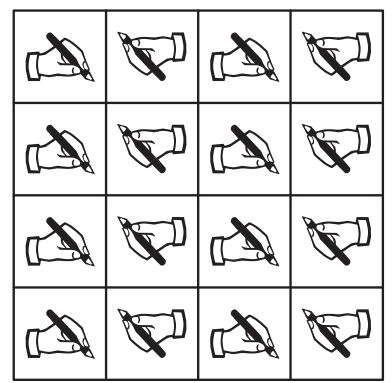

(b)

Figure 1. Examples for periodic tilings with marked squares by the transformation groups p1 (a) and p2 (b).

Now we examine a tiling (see Figure 2) in more details. We fix a tile, denoted by $P$ and consider the isometries that map $P$ onto its neighbours along the edges. We can see that the half-turn $\boldsymbol{h}_{1}$ around the midpoint of edge $A D$ maps $P$ onto $X$ and identifies $A D$ with itself changing its half-edges. In a similar way the half-turn $\boldsymbol{h}_{2}$ around the midpoint of $C D$ maps $P$ onto $Q$ and identifies $C D$ with itself changing its half-edges. Finally, we can recognize that a so-called glide reflection $g$ maps $P$ onto $T$ (while its inverse $g^{-1}$ maps $P$ onto $Z$ ) identifying the edges $A B$ and $B C$. The so-called glide axis (visualized by dotted line) and the translation part of $g$ are determined by the midpoints of $A B$ and $B C$.

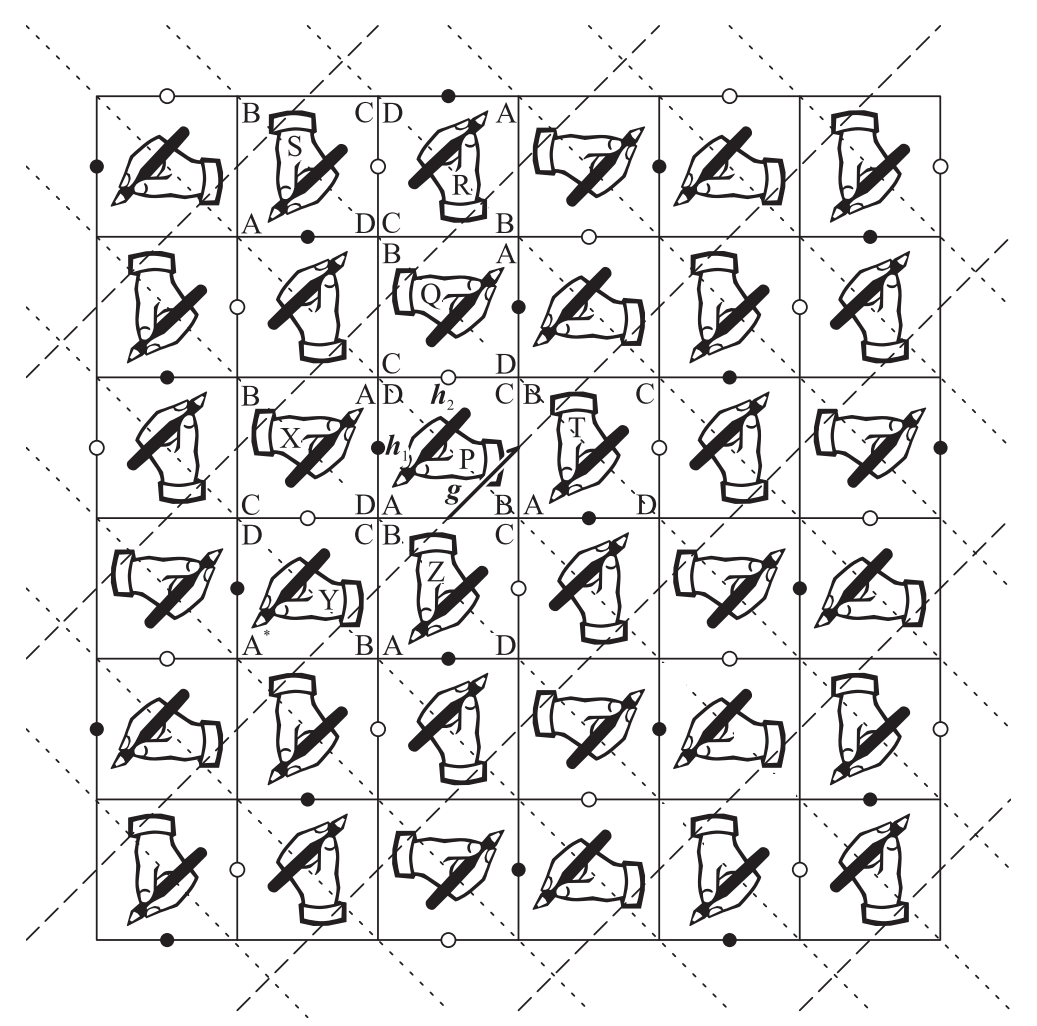

Figure 2. Fundamental tiling by crystallographic group p2gg.

We can recognize that these edge identifying transformations $\boldsymbol{h}_{1}, \boldsymbol{h}_{2}, \boldsymbol{g}, \boldsymbol{g}^{-1}$ generate the symmetry group of the tiling. Since this group, it will be denoted by $G$, acts simply transitively on the tiles, there is a one-to-one correspondence between the symmetries of $G$ and the tiles. Namely, a tile $P^{\gamma}$ 
belongs to the transformation $\gamma \in G$ that maps $P$ onto $P^{\gamma}$. $P$ itself (chosen arbitrarily) belongs to the identity transformation denoted by $\mathbf{1}$. Moreover, another arbitrary tile $L$ can be reached from $P$ through finitely many tiles that join together along edges. In this way the composition of the corresponding edge identifying transformations maps $P$ onto $L$. For example, the tile $S$ (in Figure 2 ) can be reached from $P$ through $Q$ and $R$ crossing the edges $C D, A B$ and $C D$ again. So we obtain that

$$
Q=P^{h_{2}}, \quad R=P^{g^{-1} h_{2}}, \quad S=P^{h_{2} g^{-1} h_{2}} .
$$

This formal opposite order above is a consequence, since the transformation, mapping $R$ onto $S$ through the image of $C D$, is not $\boldsymbol{h}_{2}$, as at $P$, but its so-called conjugate by $\left(\boldsymbol{g}^{-1} \boldsymbol{h}_{2}\right)$. Namely, $R$ is mapped onto $P$ by $\left(\boldsymbol{g}^{-1} \boldsymbol{h}_{2}\right)^{-1}$, then $P$ is mapped onto $Q$ by $\boldsymbol{h}_{2}$ through $C D$, then $Q$ is mapped onto $S$ by $\boldsymbol{g}^{-1} \boldsymbol{h}_{2}$ (as $P$ is mapped onto $R)$. So finally, $R$ is mapped onto $S$ by the so-called conjugate $\left(\boldsymbol{g}^{-1} \boldsymbol{h}_{2}\right)^{-1} \boldsymbol{h}_{2}\left(\boldsymbol{g}^{-1} \boldsymbol{h}_{2}\right)$. Thus,

$$
S=\left(P^{g^{-1} h_{2}}\right)^{\left(g^{-1} h_{2}\right)^{-1} h_{2}\left(g^{-1} h_{2}\right)}=P^{h_{2}\left(g^{-1} h_{2}\right)}
$$

as desired above.

In a similar way, starting from $P$ through some tiles in a cycle we can return to $P$. Then the composition of the corresponding identifying transformations is the identity, so we get a relation between the generators. Specifically, tiling around the vertices of $P$, the so-called Poincare algorithm yields the defining relations and so determines the symmetry group. In our case (in Figure 2) through the tiles $P, X, Y, Z$ we tile around the vertex $A$ of $P$. Then we obtain that

$$
X=P^{h_{1}}, \quad Y=P^{h_{2} h_{1}}, \quad Z=P^{g h_{2} h_{1}}, \quad P=P^{g^{2} h_{2} h_{1}},
$$

that means $\mathbf{1}=\boldsymbol{g}^{2} \boldsymbol{h}_{2} \boldsymbol{h}_{1}$. We can also see that in this procedure each vertex of $P$ has been mapped onto $A$, so the Poincaré algorithm gives only one relation now. Moreover, we have to take into consideration that the procedure does not produce the relations $\boldsymbol{h}_{1}^{2}=\boldsymbol{h}_{2}^{2}=\mathbf{1}$ to the involutive (involutory) generators, so these ones must be added to the previous relation(s). Thus, we get the symmetry group $G$ by generators and defining relations:

$$
\boldsymbol{G}=\left\{\boldsymbol{h}_{1}, \boldsymbol{h}_{2}, \boldsymbol{g} \mid \mathbf{1}=\boldsymbol{h}_{1}^{2}=\boldsymbol{h}_{2}^{2}=\boldsymbol{g}^{2} \boldsymbol{h}_{2} \boldsymbol{h}_{1}\right\} .
$$

Finally, we can find this group in the International Tables for Crystallography [2]: $G=$ p2gg (No. 8).

It is important to remark that the Poincaré algorithm can also be implemented clearly in a combinatorial way on the incidence structure of a tile (fundamental domain) equipped with edge identification (Figure 3), as we will see in Section 4. In this case we obtain so-called cycle transformations that will be rotations around the starting vertex in a metric realization. Thus an identification determines an appropriate transformation group if the angles of these rotations are natural divisors of the complete angle. Investigating regular $\{p, q\}$ tilings this just means that the so-called word length of any cycle transformation must be a divisor of $q$.

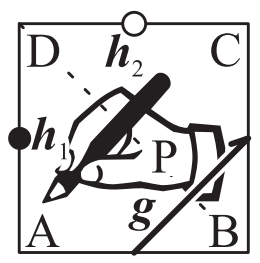

Figure 3. A fundamental domain of p2gg. 


\section{Algorithm for Finding Regular Fundamental Tilings in the Plane}

We can develop a combinatorial algorithm for finding the symmetry groups of a regular tiling. We consider henceforward the Euclidean square tiling $\{4,4\}$ and take a square from it. First of all, we look for the essentially different pairings of its edges. In a pairing an edge is paired with another one or with itself. Two pairings are said to be equivariant (essentially non-different) if there exists a symmetry of the square that maps the first pairing onto the second one. In Figure 4 the first column symbolically shows the five possibilities: each edge is paired with itself, two opposite edges form a pair and the other two ones are paired with themselves, and so on.

Then, in the second step we identify the paired edges forming edge identifications from the pairings and list the non-equivariant ones. If an edge is paired with itself then it can be identified by a half turn around its midpoint transposing its half edges or by reflection in the straight line of the edge fixing its each point. Moreover, if two opposite edges form the pair then they can be identified by a translation or by a glide reflection and its inverse whose common glide axis joins the midpoints of edges and the translation part is also determined by these two points. Finally, if two neighbouring edges form the pair then they can be identified by a quarter turn and its inverse around the common vertex or by a glide reflection and its inverse similarly to the previous case (as we have seen also in our introductory example).

Then applying the (combinatorial) Poincaré algorithm we examine each edge identification, whether it generates a symmetry group acting simply transitively on the tiling. Namely, we form the cycle transformations and check whether their word lengths are divisor of $q=4$. If so, then we can create also the defining relations, and we obtain the symmetry group. This group is a crystallographic group of the Euclidean plane, so it can be found in [2]. However, if the word length of a cycle transformation is not a divisor of 4 , then the given edge identification does not generate an appropriate group.

Finally, we can summarize our result in the following theorem. The square has 24 non-equivariant edge identifications. Among them there are 16 ones determining fundamental tilings of the Euclidean plane. These edge identifications generate 11 non-isomorphic plane crystallographic groups as Figure 4 shows by their names.

It is important to note that the edge identifications which generate fixed point free groups (p1 or pg) lead to 2-dimensional Euclidean manifolds (in the 4th and 5th rows of Figure 4). Deforming the square we glue its sides together as the identified directed edges prescribe. In this way the edge identification, which generates $\mathbf{p} \mathbf{1}$, determines the torus surface as orientable 2-manifolds, while the other two ones generating pg determine the Klein bottle as non-orientable 2-manifolds. The above gluing is logical, i.e., with the Euclidean plane $\left(E^{2}\right)$ metric.

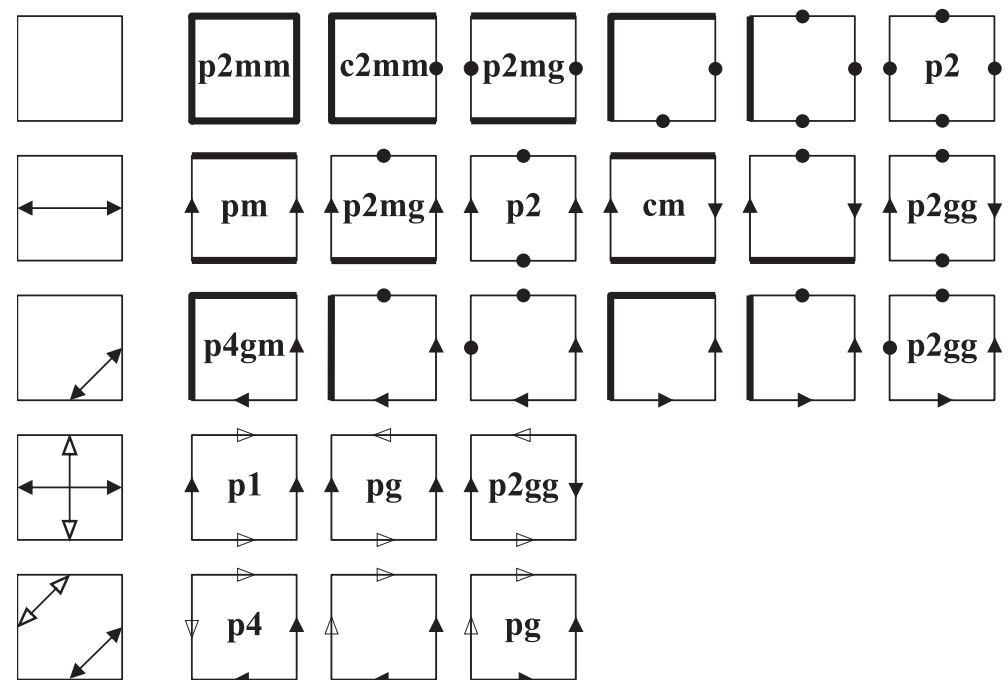

Figure 4. Edge pairings and edge identifications of the square. 


\section{Further Results in the Euclidean, Spherical and Hyperbolic Planes}

In a similar way we can examine the regular triangle tiling $\{3,6\}$ and the regular hexagon tiling $\{6,3\}$ of the Euclidean plane. Enumerating the non-equivariant edge pairings and edge identifications we form the cycle transformations and check whether their every word length is a divisor of 6 or 3 , respectively. The appropriate identifications can be seen in Figure 5 where we give also the generated crystallographic groups.

Our method enables us to examine the regular fundamental tilings of the spherical or hyperbolic plane too. The 5 regular tilings of the spherical plane $S^{2}\{3,3\},\{3,4\},\{4,3\},\{3,5\},\{5,3\}$ can be derived from the Platonic solids mapping them to a concentric sphere from the common centre. Looking for the non-equivariant edge identifications of the triangle, the square and the pentagon and applying the Poincare algorithm we can find the discrete transformation groups that act simply transitively on these tilings. Of course, each of them is a subgroup of the symmetry group of the corresponding Platonic solid. In Figure 6 we can see the appropriate edge identifications and the generated groups with their crystallographic (point group) name.

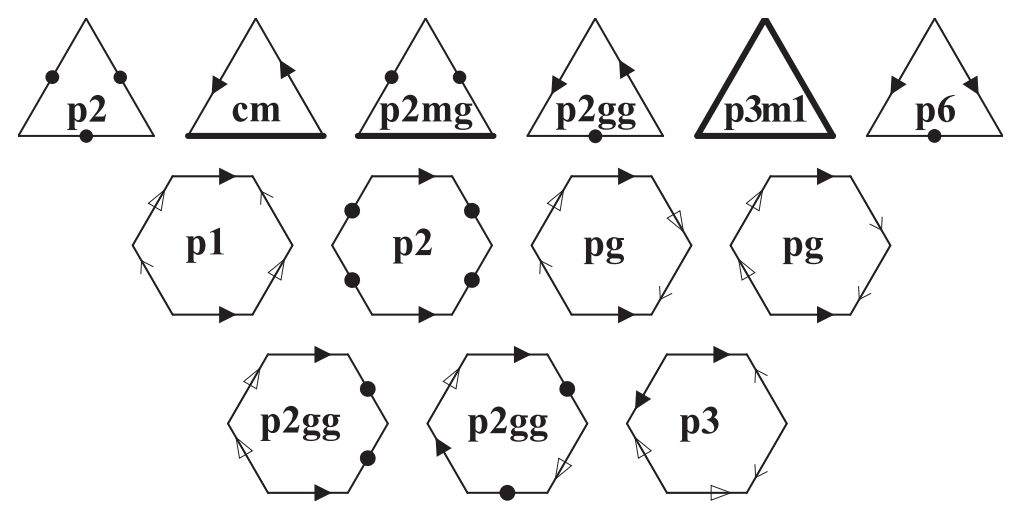

Figure 5. Edge identifications of the regular triangle and the regular hexagon generating Euclidean crystallographic groups.
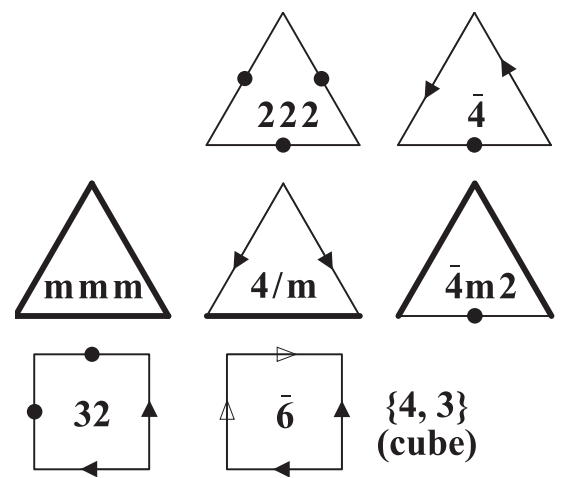

$\{4,3\}$ (cube)

\section{$\{3,3\}$}

(tetrahedron)

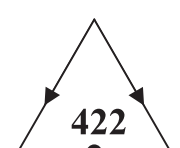

$\{3,4\}$

(octahedron)

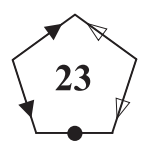

$\{5,3\}$

Figure 6. Edge identifications of the regular triangles, the square and the regular pentagon, generating fundamental spherical tilings.

The further infinitely many regular tilings $\{p, q\}$ can be realized in the Bolyai-Lobachevsky hyperbolic plane denoted by $\boldsymbol{H}^{2}$. The symmetry groups will also be infinite. The survey of discrete plane transformation groups, especially of $\boldsymbol{H}^{2}$ and their fundamental domains have already been algorithmically described after a long process from H. Poincaré to A.M. Macbeath furthermore to Z. Lučić, E. Molnár and N. Vasiljević [3]. This "old-new" paper gives the background of the topic and an algorithm, implemented also to computer (program COMCLASS) for determining all (combinatorially different) fundamental domains to a given plane group, characterized by so-called signature. The algorithm is of super-exponential complexity, but for simple signatures 
(e.g., spherical and Euclidean plane groups) it produces the classical results; also new results for other simple parameters.

In Table 1 we summarize our results for $p \leq 10, q \leq 12$. Here we emphasize only one interesting result for the self-dual tiling $\{6,6\}$. Among the 185 different edge identifications of the hexagon there are 61 ones creating the tiling $\{6,6\}$. Among these 61 identifications there are 8 ones generating fixed point free equivariant (so isomorphic) symmetry groups. These 8 identifications lead to the so-called connected sum (union) of three projective planes.

Table 1. Prn: the number of different edge pairings; Idn: the number of different edge identifications; S: spherical, E: Euclidean, (...): fixed point free.

\begin{tabular}{|c|c|c|c|c|c|c|c|c|c|c|c|c|}
\hline \multicolumn{3}{|c|}{$p, q$} & \multirow{2}{*}{3} & \multirow{2}{*}{4} & \multirow{2}{*}{5} & \multirow{2}{*}{6} & \multirow{2}{*}{7} & \multirow{2}{*}{8} & \multirow{2}{*}{9} & \multirow{2}{*}{10} & \multirow{2}{*}{11} & \multirow{2}{*}{12} \\
\hline$p$ & prn & idn ${ }^{9}$ & & & & & & & & & & \\
\hline 3 & 2 & 8 & $\mathrm{~S}[\mathrm{Tetra}] 2$ & S[Octa] 4 & $\mathrm{~S}[\mathrm{c} \cos \mathrm{a}]$ & E 6 & 0 & 4 & 2 & 2 & 0 & 8 \\
\hline 4 & 5 & 24 & $\mathrm{~S}[\text { Cube }]_{2}$ & $\mathrm{E} 16^{(3)}$ & 0 & 9 & 0 & 19 & 2 & 4 & 0 & 21 \\
\hline 5 & 6 & 52 & $\mathrm{~S}[$ Dod $] 1$ & 13 & 7 & 12 & 0 & 20 & 1 & 18 & 0 & 29 \\
\hline 6 & 17 & 185 & E $7^{(3)}$ & 39 & 9 & $61^{(8)}$ & 0 & 67 & 7 & 30 & 0 & 131 \\
\hline 7 & 27 & 578 & 3 & 47 & 6 & 71 & 45 & 105 & 3 & 68 & 0 & 251 \\
\hline 8 & 83 & 2412 & 5 & $162^{(10)}$ & 4 & 214 & 76 & $624^{(51)}$ & 5 & 106 & 0 & 872 \\
\hline 9 & 185 & 10082 & 11 & 226 & 1 & 626 & 63 & 1174 & 537 & 190 & 0 & 2864 \\
\hline 10 & 608 & 48458 & 18 & 658 & $257^{(65}$ & 1514 & 39 & 4658 & 1078 & $4080^{(473)}$ & 0 & 10162 \\
\hline
\end{tabular}

\section{Generalization of the Algorithm for 3-Dimensional Space}

The general algorithm works on the incidence structure of a $d$-dimensional polyhedron $P$ (see $[4,5])$. However, it can be shown that the complexity of this algorithm is super exponential on dimensions. The most important results refer to the 3-dimensional polyhedra having not too many faces. Thus in this section we will concentrate on $d=3$ (see also [6]).

Then the incidence structure of $P$ can be described by the set of so-called flags $Z \subseteq V \times E \times F$ where $V, E, F$ are the set of vertices, edges, faces of $P$, respectively. An ordered 3-tuple $z=(v, e, f)$ belongs to $Z(v \in V, e \in E, f \in F)$ if and only if $v, e$ and $f$ join together on the surface of $P$. If $f$ is a given face of $P$ then $\boldsymbol{Z}_{f}$ denotes the set of its flags whose face component is just $f$. Similarly, we can also speak about the flags of a given edge or a given vertex.

Two flags $z_{1}=\left(v_{1}, e_{1}, f_{1}\right)$ and $z_{2}=\left(v_{2}, e_{2}, f_{2}\right)$ are said to be vertex-adjacent if $v_{1} \neq v_{2}$ but $e_{1}=e_{2}$ and $f_{1}=f_{2}$. Similarly, we can also define the edge- and face-adjacency of two flags. We assume that the flag structure of $P$ is strictly connected. This means that each flag has exactly one face-, edge-, and vertex-adjacent flag in $\boldsymbol{Z}$, furthermore, if $\boldsymbol{a}$ and $\boldsymbol{b}$ are two different flags in $\boldsymbol{Z}$ than there is a finite sequence $\boldsymbol{a}=z_{1}, z_{2}, \ldots, z_{n}=\boldsymbol{b}$ of flags from $\boldsymbol{a}$ to $\boldsymbol{b}$ so that each flag in the sequence has the common components of $\boldsymbol{a}$ and $\boldsymbol{b}$ moreover $z_{i}$ and $z_{i+1}$ are face-, edge- or vertex-adjacent flags $(i=1,2, \ldots, n-1)$. Of course, if $P$ is a regular polyhedron, then this assumption holds automatically.

We can also speak about the incidence preserving maps of the subsets of $\boldsymbol{Z}$. For example the bijective map $\alpha: \boldsymbol{Z} \rightarrow \boldsymbol{Z}$ is an automorphism of $P$ if $\alpha$ preserves the vertex-, edge- and face-adjacencies. In a similar way, the bijective map $\alpha_{f}: Z_{f} \rightarrow Z_{f}$ is an automorphism of the face $f$ if $\alpha_{f}$ preserves the vertex- and edge-adjacencies. Moreover, the bijective map $\varphi: \boldsymbol{Z}_{g} \rightarrow \boldsymbol{Z}_{h}$ is an isomorphism of a face $g$ onto another face $h$ if $\varphi$ preserves the vertex-and edge-adjacencies. In this way we can arrange the faces of $P$ into isomorphism classes.

It is easy to see that the automorphism group of a (combinatorial) $n$-gon is the dihedral group $\boldsymbol{D}_{n}$, moreover a $k$-gon and an $\ell$-gon are isomorphic if and only if $k=\ell$. If $P$ is regular, then all of its faces are regular $n$-gons $(n=3,4,5)$, thus the faces form only one isomorphism class. We can also recognize that the automorphism group of a regular polyhedron is isomorphic with its (metric) symmetry group.

We can also define the so called face mappings as bijective maps $\boldsymbol{Z} \rightarrow \boldsymbol{Z}$ that preserve the vertexand edge-adjacencies (but do not preserve necessarily the face-adjacencies). Thus the restriction 
of a face mapping onto the flags of an arbitrary face is an isomorphism. We can also see that the automorphism group Aut $P$ is a subgroup of the face mapping group Fcmp $P$.

In our 2-dimensional example in Section 2 first we have looked for the non-equivariant (essentially different) pairings and identifications of edges of the fundamental polygon. Now, considering a 3-dimensional fundamental polyhedron $P$, we have to search for different face pairings and face identifications.

A face pairing contains disjoint pairs of faces so that each face occurs in exactly one pair but certain faces can also be paired with itself. During the enumeration of the possible pairings, we have to check whether the actual one is different from all the previous ones. Two pairings are equivariant if there is an automorphism of $P$ that maps the first pairing onto the second one.

Then we equip the pairings with face identifying transformations. This means that we give an isomorphism $\varphi_{g}: \boldsymbol{Z}_{g} \rightarrow \boldsymbol{Z}_{h}$ and its inverse $\bar{\varphi}_{h}: \boldsymbol{Z}_{h} \rightarrow \boldsymbol{Z}_{g}\left(\bar{\varphi}_{h}=\varphi_{g}^{-1}\right)$ to each pair $(g, h)$, where $g \neq h$, or an involutive automorphism $\alpha_{f}: \boldsymbol{Z}_{f} \rightarrow \boldsymbol{Z}_{f}\left(\alpha_{f}^{-1}=\alpha_{f}\right)$ to each pair $(f, f)$ where a face is paired with itself. We can imagine that in the periodic face-to-face tiling the isomorphism $\varphi_{g}$ prescribes how the fundamental polyhedron $P$ is mapped (by the corresponding isometry $\varphi_{g}^{\star}$ ) onto its neighbour along $h$ mapping $g$ onto $h$, and how $P$ is mapped (by $\bar{\varphi}_{h}^{\star}$ ) onto its neighbour along $g$ mapping $h$ onto $g$.

Considering the identifying isomorphisms and their inverses (moreover the identifying involutive automorphisms too) we can also recognize that their union is just an involutive face mapping $\delta$ $\left(\delta \in \mathrm{Fcmp} P, \delta^{-1}=\delta\right)$. In this way the face identifications are just the involutive elements of Fcmp $P$, moreover two face identifications $\delta_{1}$ and $\delta_{2}$ are equivariant if and only if there is an automorphism $\alpha \in$ Aut $P$ mapping $\delta_{1}$ onto $\delta_{2}$, namely $\delta_{2}=\alpha^{-1} \delta_{1} \alpha$.

In Section 2 we have also seen how the Poincaré algorithm forms the defining relations (more precisely the cycle transformations) to a given system of edge identifying generators. Now the generators are given by a face identification $\delta \in \operatorname{Fcmp} P\left(\delta^{-1}=\delta\right)$. We choose an edge $e_{1}$ and a flag $z_{1}=\left(v_{1}, e_{1}, f_{1}\right)$ joining it. Then we consider the face component $f_{1}$ of $z_{1}$ and write the name $\varphi_{1}^{\star}$ of the corresponding transformation belonging to $\varphi_{1}=\left.\delta\right|_{f_{1}}$ (the restriction of $\delta$ onto the flags of $f_{1}$ ). The next flag $z_{2}=\left(v_{2}, e_{2}, f_{2}\right)$ will be the face-adjacent flag of $z_{1}^{\delta}$. Then we write the name of the second transformation belonging to $\varphi_{2}=\left.\delta\right|_{f_{2}}$, thus we obtain the composition $\varphi_{1}^{\star} \varphi_{2}^{\star}$. We continue this method until in the $n^{\text {th }}$ step we get back the starting flag $z_{1}$ as the face-adjacent flag of $z_{n}^{\delta}$. Then we obtain the cycle transformation $\varphi_{1}^{\star} \varphi_{2}^{\star} \cdots \varphi_{n}^{\star}$ which could be a defining relation with an appropriate exponent $k$ : $\left(\varphi_{1}^{\star} \varphi_{2}^{\star} \cdots \varphi_{n}^{\star}\right)^{k}=1$. Starting with an edge not appeared among $e_{1}, e_{1}, \ldots, e_{n}$, we can find another cycle transformation, and so on until each edge occurs in a cycle.

Examining the complexity of the algorithm, it can be established that the most calculations are necessary to enumerate the essentially different face identifications. Deeper examination shows that their number grows exponentially with the number of faces. For example the number of different face identifications of the tetrahedron, the cube and the octahedron are $64,8260,92,340$, respectively. Unfortunately these numbers are not known for the dodecahedron and the icosahedron exactly now, but their orders of magnitude are $10^{10}$ and $10^{17}$, respectively.

\section{Results in 3-Dimensional Spaces of Constant Curvature}

A regular 3-dimensional tiling can be described by its Schläfli symbol $\{p, q, r\}$ which signifies that the tiles are congruent regular polyhedra $\{p, q\}$ so that in the tiling there are exactly $r$ tiles around each edge. The vertex figures $\{q, r\}$ are also regular polyhedra (or regular horospherical tilings if the vertices are ideal points on the absolute of the hyperbolic space). In the Euclidean space the only regular tiling is the cube tiling $\{4,3,4\}$. The regular tilings of the spherical space can be derived from the regular 4-dimensional polyhedra projecting them onto the concentric 4-dimensional sphere. In this manner there are 6 (finite) regular spherical tilings: $\{3,3,3\},\{3,3,4\},\{3,3,5\}$ with 3 -faces (or cells) 5 , 16,600 tetrahedra, respectively, $\{3,4,3\}$ has 24 octahedra as 3 -faces, $\{4,3,3\}$ has 8 cubes and $\{5,3,3\}$ has 120 dodecahedra as 3 -faces. In the hyperbolic space there are 4 regular tilings with proper (inner) vertices: $\{3,5,3\}$ having icosahedra as 3 -tiles, $\{4,3,5\}$ with cubes, $\{5,3,4\},\{5,3,5\}$ with dodecahedra. 
Moreover, there are 4 regular tilings with ideal vertices on the absolute: $\{3,3,6\}$ with tetrahedra, $\{3,4,4\}$ with octahedra, $\{4,3,6\}$ with cubes and $\{5,3,6\}$ with dodecahedra.

Related to the problem of square tiling in Section 1.1 first we consider the cube and look for the pairings of its 6 faces. So we get 10 different possibilities. Adding identifying transformations to them (combinatorially), finally we get 8260 essentially different face identifications. Applying the Poincaré algorithm we can examine which ones generate discrete transformation groups acting simply transitively on tilings $\{4,3, r\}$, where $r=3,4,5,6$. In case of $r=4$ we have to choose those identifications where the word lengths of cycle transformations are 1, 2 or 4, thus, with corresponding exponent $(4,2$ or 1 , respectively) we obtain defining relations of word length 4 . Then on the basis of geometric properties of the identifying generators we look up the group in the International Tables [2]. In this way we get the following result: the cube has 298 face identifications generating 130 non-isomorphic Euclidean crystallographic groups. Among these identifications there are $6+12$ ones that generate $5+4$ fixed point free (orientable + non-orientable) space groups determining Euclidean 3-manifolds. The complete list of the 298 face identifications and the 130 space groups can be found in [7]. Two of them can be seen in Figure 7. In the first case screw motions of half turn with parallel and skew axes identify the faces of the cube generating the crystallographic group $\mathbf{P} \mathbf{2}_{\mathbf{1}} \mathbf{2}_{\mathbf{1}} \mathbf{2}_{\mathbf{1}}$ (No. 19) while in the second case screw motions of third turn with parallel axes generate the space group $\mathbf{P} 3_{1}$ (No. 144). Both of them are fixed point free so they determine orientable Euclidean space forms.

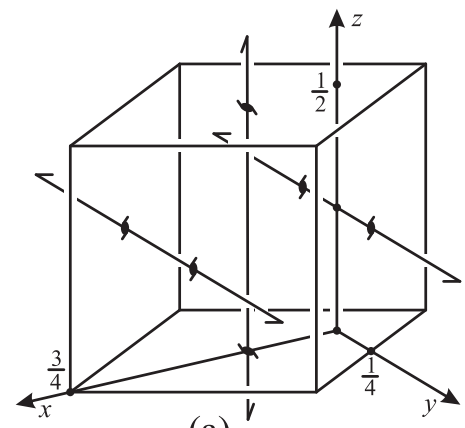

(a)

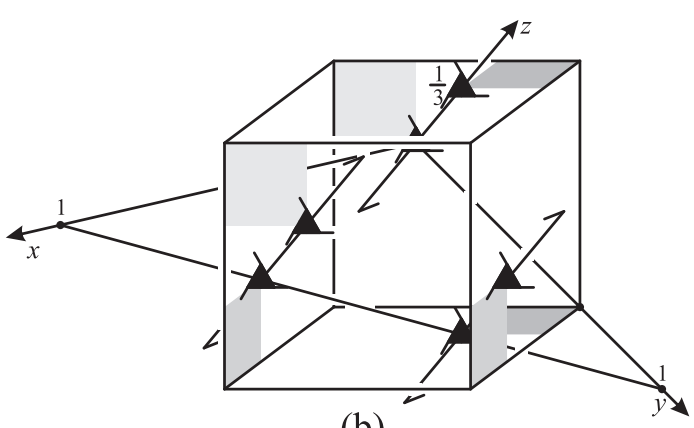

(b)

Figure 7. Two examples for face identifications of the cube generating fixed point free fundamental cube tilings in the Euclidean space. The generated groups are $\mathbf{P} \mathbf{2}_{\mathbf{1}} \mathbf{2}_{\mathbf{1}} \mathbf{2}_{\mathbf{1}}(\mathbf{a})$ and $\mathbf{P} \mathbf{3}_{\mathbf{1}}(\mathbf{b})$.

In the cube tiling $\{4,3,3\}$ of the spherical space each edge is surrounded by 3 cubes. So we have to look up those face identifications among the 8260 ones, which yield to cycle transformations of word length 1 or 3 by the Poincare algorithm. Since the tiling contains 8 cubes, we obtain groups of order 8 . Finally, we obtain that the cube has 7 face identifications generating 5 non-isomorphic space groups. Two of them are fixed point free yielding orientable spherical space forms. In Figure 8 we can see the last two ones, where screw motions identify the faces. The generated groups are isomorphic with the cyclic group $\mathbf{Z}_{8}$ and the quaternion group $\mathbf{Q}$ [8], respectively.

Considering the hyperbolic tiling $\{4,3,5\}$ we obtain the following surprising result: among the 8260 face identifications of the cube there is only one identification that generates the hyperbolic space group $\mathrm{G}^{(5)}$ acting simply transitively on the tiles of $\{4,3,5\}$. The identifying generators are two rotations of order 5 and a screw motion, as we can see in Figure 9a (the first identification) $[9,10]$. Since (for example) the points of rotation axes are fixed, this identification does not determine a space form. 


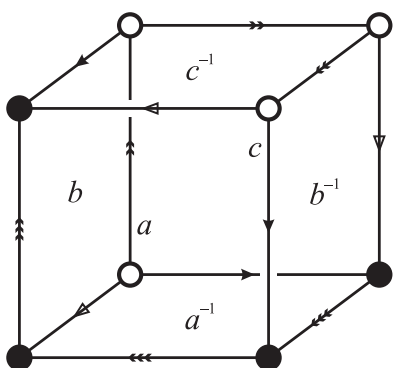

(a)

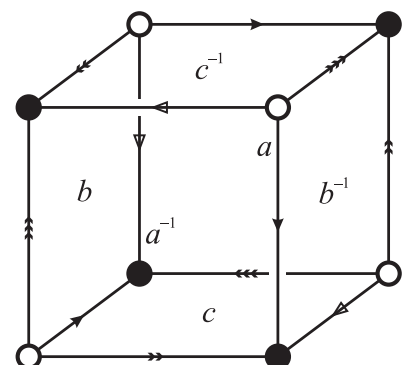

(b)

Figure 8. The two face identifications of the cube determining fixed point free spherical tilings. The generated groups are $\mathbf{G}_{4}^{(3)} \cong \mathbf{Z}_{\mathbf{8}}(\mathbf{a})$ and $\mathrm{G}_{\mathbf{7}}^{(3)} \cong \mathbf{Q}(\mathbf{b})$.

Finally, in the hyperbolic tiling $\{4,3,6\}$ each edge is surrounded by six cubes, moreover the vertices are ideal points on the absolute (so they not belong to the space). The cube has 203 face identifications generating hyperbolic space groups. Among them there are $3+8$ ones that generate fixed point free groups determining at least $2+7$ non-compact (but having finite volume) orientable and non-orientable hyperbolic manifolds [10-12]. In Figure 9 the second and the third face identifications present both cases with an example, respectively.

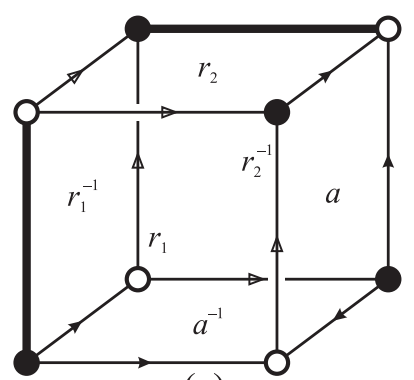

(a)

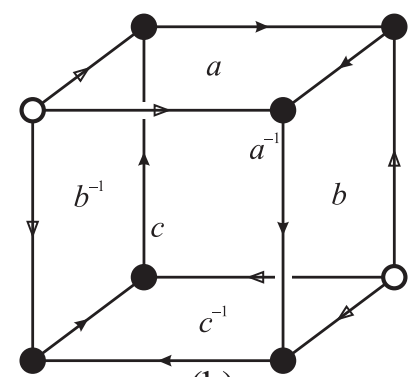

(b)

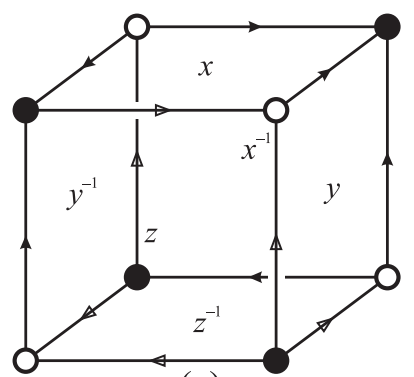

(c)

Figure 9. The only face identification of the cube determining the hyperbolic fundamental tiling $\{4,3,5\}$ by the space group $G^{(5)}(\mathbf{a})$. The two additional ones generate the fixed point free hyperbolic space groups $G_{i}^{(6)}(b)$ and $G_{b}^{(6)}$ (c) to tiling $\{4,3,6\}$ [10].

Usually, in order to decide whether a face identification determines a fixed point free group, we have to examine whether the stabilizer groups of faces, edges and vertices are trivial. For example, each pair of faces must contain different faces, since if a face was paired with itself then the corresponding identifying transformation would fix (at least) one point of this face. (So the number of faces must be even.) Moreover, the number of different edges enumerated by the Poincaré algorithm forming a cycle transformation must be just $r$ (namely the number of tiles around the edges), otherwise (at least) the midpoints of the appeared edges would be fixed. (This also means that $r$ must be a divisor of the number of edges of the polyhedron.) Finally, if the vertices are proper (inner) points of the space, we have to consider the classes of vertices determined by the face identification. Two vertices belong to the same class if there is a sequence of the vertices from the first one to the second one in which an arbitrary vertex is mapped onto the next one by the identification. Then the number of vertices in an arbitrary class must be equal with the number of tiles surrounding the vertices in the given tiling $\{p, q, r\}$.

However, if the vertices of a hyperbolic tiling are ideal points on the absolute, then the vertex stabilizer subgroups do not have to be trivial. Intersecting the tiling with a horosphere centred at a vertex we obtain a (regular) horospherical polygon tiling. From the neighbouring polygons belonging to different vertices from the given vertex class we can glue the fundamental domain of the vertex stabilizer subgroup. This group and so its restriction to the horosphere is also fixed point free. Since the 
horosphere is isometric with the Euclidean plane, the stabilizer must be isomorphic with one of the fix point free plane crystallographic group $\mathbf{p} 1$ or $\mathbf{p g}$. The vertex classes and the corresponding stabilizer groups form the so-called cusp structure which play an important role to decide whether two face identifications generate isomorphic space groups or not. If the cusp structures are different, then the groups cannot be isomorphic.

If a face identification generates a fixed point free space group $G$ then the fundamental group of the determined manifold is also $G$, while the first homology group $H_{1}=G /[G, G]$ is the commutator factor group of $G$. This group can be compute easily from the defining relations of $G$. The first homology group is also an important tool in the examination of isomorphism. In this way we can recognise if two infinite groups are not isomorphic. However, usually the question of isomorphism of infinite groups is difficult. This is the reason why we can say only a lower limit for the number of generated groups in certain cases.

In Table 2 we summarise the results related to the regular tilings of 3-dimensional spaces of constant curvature. The classification of (not only regular) simplex tilings can be found in [6,13-15]. In $[14,15]$ the examination was extended also to the other homogeneous 3-spaces. The octahedron manifolds were discovered by J. M. Montesinos [8], B. Everitt [16] and M. Šarać, while the 3 icosahedron manifolds were enumerated by L. A. Best [9]. The spherical dodecahedron spaces were discovered by H. Poincaré, P. J. Lorimer [17] and B. Everitt [16].

Table 2. Face id: the number of face identifications generating space groups. Fixed point free: the number of face identifications generating orientation preserving and orientation reversing fixed point free groups. Manifold: the number of determined orientable and non-orientable manifolds ( $\operatorname{star}\left(^{*}\right)$ means that the number is only a proved lower limit).

\begin{tabular}{|c|c|c|c|c|c|c|c|}
\hline Space & Tiling & $\begin{array}{c}\text { Fundamental } \\
\text { domain }\end{array}$ & Face id. & $\begin{array}{c}\text { Fixed point } \\
\text { free }\end{array}$ & \multicolumn{2}{|c|}{ Manifold } \\
\hline $\boldsymbol{S}^{3}$ & $\{3,3,3\}$ & tetrahedron & 1 & 1 & 0 & 1 & 0 \\
$\boldsymbol{S}^{3}$ & $\{3,3,4\}$ & tetrahedron & 14 & - & - & - & - \\
$\boldsymbol{S}^{3}$ & $\{3,3,5\}$ & tetrahedron & 1 & - & - & - & - \\
$\boldsymbol{S}^{3}$ & $\{3,4,3\}$ & octahedron & 4 & 3 & 0 & 3 & 0 \\
$\boldsymbol{S}^{3}$ & $\{4,3,3\}$ & cube & 7 & 2 & 0 & 2 & 0 \\
$\boldsymbol{S}^{3}$ & $\{5,3,3\}$ & dodecahedron & $?$ & 2 & 0 & 2 & 0 \\
\hline $\boldsymbol{E}^{3}$ & $\{4,3,4\}$ & cube & 298 & 6 & 12 & 5 & 4 \\
\hline $\boldsymbol{H}^{3}$ & $\{3,3,6\}$ & tetrahedron & 9 & 0 & 1 & 0 & 1 \\
$\boldsymbol{H}^{3}$ & $\{3,4,4\}$ & octahedron & 368 & 2 & 11 & $1^{*}$ & $6^{*}$ \\
$\boldsymbol{H}^{3}$ & $\{3,5,3\}$ & icosahedron & $?$ & 3 & $?$ & 3 & $?$ \\
$\boldsymbol{H}^{3}$ & $\{4,3,5\}$ & cube & 1 & - & - & - & - \\
$\boldsymbol{H}^{3}$ & $\{4,3,6\}$ & cube & 203 & 3 & 8 & $2^{*}$ & $7^{*}$ \\
$\boldsymbol{H}^{3}$ & $\{5,3,4\}$ & dodecahedron & $?$ & - & - & - & - \\
$\boldsymbol{H}^{3}$ & $\{5,3,5\}$ & dodecahedron & $?$ & 12 & 0 & $7^{*}$ & 0 \\
$\boldsymbol{H}^{3}$ & $\{5,3,6\}$ & dodecahedron & $?$ & 10 & 68 & $7^{*}$ & $31^{*}$ \\
\hline
\end{tabular}

In Table 2 we can see that the dodecahedron has 12 essentially different face identifications which generate fixed point free orientation preserving transformation groups acting simply transitively on the $\{5,3,5\}$ tiling of the hyperbolic space. These face identifications determine compact orientable hyperbolic manifolds. Considering their first homology groups we obtain that there are at least 7 different ones among them. Our algorithm also shows that compact non-orientable hyperbolic dodecahedron manifolds do not exist. The first compact hyperbolic dodecahedron manifold was discovered by C. Weber and H. Seifert [18]. Then L. A. Best listed 7 more ones in [9], however 3 of them were not different. Finally, the complete list was created by our algorithm in [19], determining at least 3 new manifolds according to their first homology groups. 
In Figure 10 we can see one of these face identifications. The orientation preserving face identifying generators $\boldsymbol{a}, \boldsymbol{b}, \boldsymbol{c}, \boldsymbol{d}, \boldsymbol{e}, f$ map the faces $a^{-1}, b^{-1}, c^{-1}, d^{-1}, e^{-1}, f^{-1}$ onto $a, b, c, d, e, f$ mapping the dodecahedron onto its neighbours along $a, b, c, d, e, f$, respectively. Similarly, the inverses of the generators $\boldsymbol{a}^{-1}, \boldsymbol{b}^{-1}, \boldsymbol{c}^{-1}, \boldsymbol{d}^{-1}, \boldsymbol{e}^{-1}, f^{-1}$ map the faces $a, b, c, d, e, f$ onto $a^{-1}, b^{-1}, c^{-1}, d^{-1}, e^{-1}$, $f^{-1}$ mapping the dodecahedron onto its neighbours along $a^{-1}, b^{-1}, c^{-1}, d^{-1}, e^{-1}, f^{-1}$, respectively. The Poincaré algorithm yields 6 defining relations of word length 5 forming 6 classes of directed edges. In Figure 10 the edges belonging to common class are denoted by the same type of arrows. According to the condition that there are 5 dodecahedra around the edges in tiling $\{5,3,5\}$, each edge class contains exactly 5 directed edges which are mapped onto each other during the transformations tile around one of them. We can also recognize that the 20 vertices belong to only one class as the face identification prescribes, according to the icosahedral vertex figure of $\{5,3,5\}$. In this way fixed points cannot be appeared. The dodecahedron as a tile in $\{5,3,5\}$ is compact, moreover the identifying transformations and so the generated group $G$ preserves the orientation. Thus the face identification determines an orientable compact manifold with fundamental group $G$. Finally, as the Abelianization of $G$ we get the first homology group $H_{1}=Z_{29}$ (the cyclic group of order 29) of the manifold. This homology group belongs to only this identification so $G$ cannot be isomorphic with the fundamental groups determined by the other identifications.
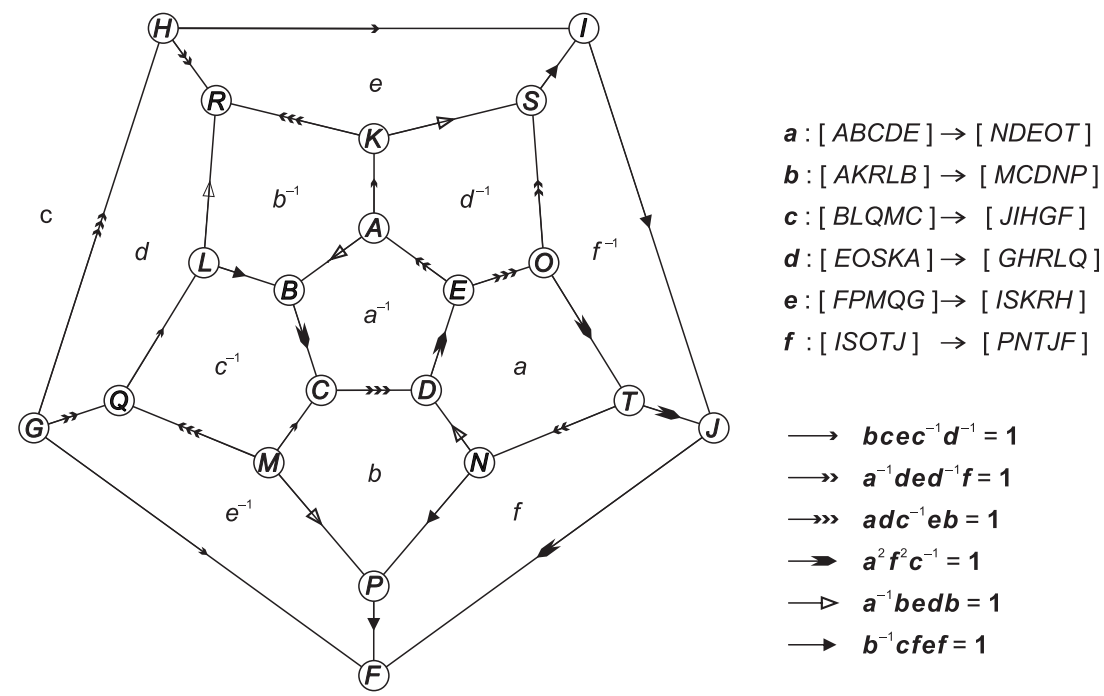

Figure 10. Face identification of the dodecahedron yielding compact hyperbolic manifold of first homology group $\mathbf{Z}_{29}$.

In the last line of Table 2 we can see the results for the hyperbolic dodecahedron tiling $\{5,3,6\}$ in which the vertices are ideal points. Among them the 10 face identifications determining orientable non-compact manifolds were discovered independently by B. Everitt [16] (clearly with algebraic tools) and by the author [19], while the 68 face identifications yielding non-orientable non-compact hyperbolic manifolds were new results in [19].

Acknowledgments: The author thanks his colleague Prof. Emil Molnár for his valuable suggestions.

Conflicts of Interest: The author declares no conflict of interest.

\section{References}

1. Molnár, E.; Prok, I.; Szirmai, J. On maximal homogeneous 3-geometries and their visualization. Universe 2017, 3, 83.

2. Hahn, T. (Ed.) International Tables for Crystallography, Volume A: Space-Group Symmetry; Springer: Berlin, Germany, 2005. 
3. Lučić, Z.; Molnár, E.; Vasiljević, N. An algorithm for classification of fundamental polygons for a plane discontinuous group. In Discrete Geometry and Symmetry; Conder, M.D.E., Deza, A., Ivič Weiss, A., Eds; Springer: Berlin, Germany, 2018.

4. Molnár, E. Polyhedron complexes with simply transitive group actions and their realizations. Acta Math. Hung. 1992, 59, 175-216.

5. Prok, I. Data structures and procedures for a polyhedron algorithm. Period. Polytech. Mech. Eng. 1992, 36, 299-316.

6. Molnár, E.; Prok, I. A polyhedron algorithm for finding space groups. In Proceedings of the Third International Conference on Engineering Graphics and Descriptive Geometry, Vienna, Austria, 11-16 July 1988.

7. Prok, I. The Euclidean space has 298 fundamental tilings with marked cubes by 130 space groups. In Colloquia Math. Soc. János Bolyai, 63. Intuitive Geometry; Böröczky, K., Fejes Tóth, G., Eds.; North-Holland Publ. Comp.: Amsterdam, The Netherlands, 1994; pp. 363-388.

8. Montesinos-Amilibia, J.M. Classical Tessellations and Three-Manifolds; Springer: Berlin, Germany, 2012.

9. Best, L.A. On torsion-free discrete subgroups of $\operatorname{PSL}(2$, C) with compact orbit space. Can. J. Math. 1971, 23, 451-460.

10. Prok, I. Fundamental tilings with marked cubes in spaces of constant curvature. Acta Math. Hung. 1996, 71, 1-14.

11. Molnár, E. Minimal presentation of the 10 compact Euclidean space forms by fundamental domains. Stud. Sci. Math. Hung. 1987, 22, 19-51.

12. Aitchison, R.; Rubinstein, J.H. An introduction to polyhedral metrics of non-positive curvature on 3-manifolds. In Geometry of Low-Dimensional Manifolds: 2; Donalds, S.K., Thomas, C.B., Eds.; Cambridge University Press: Cambridge, UK, 1990; Volume 151, pp. 127-161.

13. Molnár, E.; Prok, I. Classification of solid transitive simplex tilings in simply connected 3-spaces, Part 1. The combinatorial description by figures and tables, results in spaces of constant curvature. In Colloquia Math. Soc. János Bolyai, 63. Intuitive Geometry; Böröczky, K., Fejes Tóth, G., Eds.; North-Holland: Amsterdam, The Netherlands, 1994; pp. 311-362.

14. Molnár, E.; Prok, I.; Szirmai. J. Classification of solid transitive simplex tilings in simply connected 3-spaces, Part 2. Metric realizations of the maximal simplex tilings. Period. Math. Hung. 1997, 35, 47-94.

15. Molnár, E.; Prok, I.; Szirmai, J. Classification of tile-transitive 3-simplex tilings and their realizations in homogeneous geometries. In Non-Euclidean Geometries, János Bolyai Memorial Volume; Prékopa, A., Molnár, E., Eds.; Springer: Berlin, Germany, 2005.

16. Everitt, B. 3-manifolds from Platonic solids. Topol. Its Appl. 2004, 138, 253-263.

17. Lorimer, P.J. Four dodecahedral spaces. Pac. J. Math. 1992, 156, 329-335.

18. Weber, C.; Seifert, H. Die beiden Dodekaederräume. Math. Z. 1933, 37, 237-253.

19. Prok, I. Classification of dodecahedral space forms. Beiträge zur Algebra Geometrie 1998, 39, 497-515.

(C) 2018 by the author. Licensee MDPI, Basel, Switzerland. This article is an open access article distributed under the terms and conditions of the Creative Commons Attribution (CC BY) license (http:/ / creativecommons.org/licenses/by/4.0/). 